\title{
Social capital and Health
}

1. Social capital as a key factor to achieve better society

Akashi National College of Technology : Ishida, Yu

2. Social capital and health : experiences from Sweden

Lund University : Jan Sundquist

3. Geographic Information System and Health

Esri Japan Corporation : Tsuchida, Masayo

4. Place and people : what can we learn from the evidence?

Osaka City University : Kimura, Yoshinari

Chairpersons : Nabika, Toru : Shimane University

Kimura, Yoshinari : Osaka City University

\section{Background}

During the last decade, great efforts were taken to identify effects of social environment on health. Previous reviews defined that the social environment includes the degree and the nature of social connections between neighbors, the presence of social norms, levels of safety and violence, and various features of the social organizations. The social capital is one of the measurements of the social environment, and it has already been shown that the social capital is associated with a variety of health outcomes including physical health (e.g., self-rated health, obesity) and mental health (e.g., depression, anxiety). However, at present, it is unclear how to promote or maintain the social capital.

In addition, there is growing interest to geographic information systems (GIS) in recent years. GIS allow us to combine a variety of data, and visualize the features of each neighbourhood. Accordingly, it helps us to understand the differences of the social capital among neighbourhoods, and give a useful insight to the social capital research. We often need to know why some of neighbourhoods have high social capital. This workshop thus included four presentations: the first two were about the social capital research and the rest were about the GIS research. Our goal was to share the evidence on the association of the social capital and the health (not only the physical health but also the social welfare), and to discuss on how to promote the social capital in neighbourhoods on the basis of the geographical perspective.

\section{Overview of presentations}

(1) Social capital and health : experiences from Sweden

Jan Sundquist (Lund University)

The first speaker was Dr. Sundquist from the Lund University, Sweden. He showed empirical associations between social capital and the coronary heart disease (CHD). A follow-up study of 1,358,932 men and $1,446,747$ women aged $45-74$ years was conducted between January 1998 and December 1999. The Swedish Government-owned Census Bureau has geocoded the home addresses 
of all Swedish individuals to Small Area Market Statistics (SAMS). These SAMS were therefore used to define neighborhoods in this study. The neighbourhood-inking social capital was measured with the proportion of individuals voting in elections of the local governments at the neighbourhood level. The neighbourhood- and the individual-level factors were analysed in a multilevel fashion. The linking social capital was associated more with $\mathrm{CHD}$, one of the leading causes of death worldwide, in both men and women when compared with the factors of the individual-level; in neighbourhoods with low linking social capital, the odds ratios were $1.19(95 \%$ confidence interval $=1.14-1.24)$ and 1.29 (95\% confidence interval $=1.21$ 1.38) for men and women, respectively, after adjustment for age, country of birth, education, marital status, and housing tenure. The significant between-neighbourhood variance (i.e. the random intercept) showed significant differences in CHD incidence between neighbourhoods. Even in a relatively egalitarian society, as exemplified by the Swedish Welfare State, individual health is affected by the difference the linking social capitals between neighbourhoods. The use of linking social capitals may provide a novel conceptual advance in the research on the association between various health outcomes and the multidimensional aspects of the social capital.

(2) Social capital as a key factor to achieve better society

Ishida, Yu (Akashi National College of Technology)

Second speaker was Dr. Ishida from the Akashi National College of Technology, Japan. His presentation mainly focused on the issues of volunteering and the social capital in terms of disaster management in local so- cieties. He presented data on many for-profit organizations (FPOs) in Toyooka city who gave effort to save the residences in their communities in the time of disaster by the flood. He showed that $34.8 \%$ of the FPOs did not take daily activities preparing against disasters, although about the $40 \%$ were cooperating with their communities. In addition, methods of making agreements were not spread irrespective of both public administration and local communities. The data told us who made the corporations decide to carry out their daily activities for communities ; it was natural to interpret that "the head of a corporation" gave instructions to the employees, but it was interesting for us to see "residents in a community" affected the decision of FPOs. This indicated that FPOs would determine their activities according to requests of local communities. For instance, of $52.3 \%$ of the FPOs "participating in local fire drills", the $24.3 \%$ decided to participate on the request of the community or of residents, while the $25.0 \%$ did it by the order of the head or the director. This result suggested that leadership of the civil society could be as important in disaster management as the leadership of the local governments, that of the business unions, and financial and material supports. Besides focusing on the relations between these two players, we also need to focus on volunteering. The author examined which local area had more volunteers. The result was quite reasonable that more volunteers were seen in the area where healthier people lived. Thus, promoting health in the local area may be necessary to construct local societies that can give better disaster management.

(3) Geographic Information System and Health 
Tsuchida, Masayo (Esri Japan Corporation)

The third speaker was Ms. Tsuchida from Esri Japan Corporation, Japan. Her presentation showed us a current trend of GIS and health research. She addressed our question what is GIS? The answer was very clear, and we were able to share new knowledge as follows; the GIS integrates hardware, software, and data for capturing, managing, analyzing, and displaying all forms of geographically referenced information. Moreover, GIS allows us to view, understand, question, interpret, and visualize data in many ways that reveal relationships, patterns, and trends in the form of maps, globes, reports, and charts. The GIS helps us to answer questions and solve problems by providing the data that are quickly understood and easily shared. She also showed that the research on GIS and Health had a close and old history. Since Dr. John Snow showed a cholera map in London, 1854, GIS has been a powerful tool of the public health research, lifestyle-related diseases, infectious diseases, hospital management, ward area of marketing, and crisis management. The GIS is used in health researches around the world, including the Centers for Disease Control and Prevention and the World Health Organization. Moreover, health and human services have been developed as follows:public health, hospital and health systems, human services, managed care, academic programs and research.

(4)Place and people: what can we learn from the evidence?

Kimura, Yoshinari (Osaka City University)

The last speaker was Dr. Kimura from Osaka City University, Japan. He showed us an advantage of GIS in the health study on the basis of empirical evidences. His interest was to know where people lived (not who you were). The first example was to clarify the accessibility to emergency hospitals in a secondary-medical care sector in Shimane prefecture. He categorized areas into three; the area where people could access to the hospitals within $3 \mathrm{~min}, 10 \mathrm{~min}$, and $30 \mathrm{~min}$. transportation time to evaluate the accessibility to emergency hospitals by calculating population in that area. The western part of Shimane, such as the Oda, the Masuda and the Hamada area, had more residents living in the area with over 30 minutes of the transportation time when compared to the eastern part. In the latter part of his presentation, a research on the association between physical environment and health was also introduced. This research in a rural mountainous region in Japan showed that the distance from a population center was associated with a higher risk of hypertension. He concluded that such GIS researches in the rural medicine had just started, so that further studies were needed to expand the application.

\section{Discussion}

In the light of these presentations, active discussion was given about future directions of the social capital research. The first step is to make a map to understand the variance of social capital among areas. This is because neighbourhood in the rural region now faces to an aging depopulated society, so that there might be substantial differences of social capital among the communities in a rural region. This means that the social capital is not always a resource to promote our health. Based on shared understanding of the current situation of the social capital in a rural region, we will be able to start discussions re- 
garding how to promote the social capital and how to use its potential to maintain/improve our health. We believe that the social capital is one of the unique resources in rural regions, and it will give us a useful insight for health promotion in the near future. 